\title{
Next-to-leading Order Calculation of the Single Transverse Spin Asymmetry in the Drell-Yan Process
}

\author{
Werner Vogelsang $1, *$ and Feng Yuan ${ }^{2,3,+}$ \\ ${ }^{1}$ Physics Department, Brookhaven National Laboratory, Upton, NY 11973 \\ ${ }^{2}$ Nuclear Science Division, Lawrence Berkeley National Laboratory, Berkeley, CA 94720 \\ ${ }^{3}$ RIKEN BNL Research Center, Building 510A, \\ Brookhaven National Laboratory, Upton, NY 11973
}

(Dated: November 13, 2018)

\begin{abstract}
We calculate the next-to-leading order perturbative QCD corrections to the transverse momentum weighted single transverse spin asymmetry in Drell-Yan lepton pair production in hadronic collisions. We identify the splitting function relevant for the scale evolution of the twist-three quark-gluon correlation function. We comment on the consequences of our results for phenomenology.
\end{abstract}

*Electronic address: vogelsan@quark.phy.bnl.gov

†Electronic address: fyuan@quark.phy.bnl.gov 


\section{INTRODUCTION}

Single transverse spin asymmetries (SSAs) in high energy hadronic reactions continue to attract much theoretical and experimental interest. They are defined as differences of cross sections when one of the initial hadrons' transverse spin is flipped, divided by the sum: $A_{N} \sim\left(d \sigma\left(S_{\perp}\right)-d \sigma\left(-S_{\perp}\right)\right) /\left(d \sigma\left(S_{\perp}\right)+d \sigma\left(-S_{\perp}\right)\right)$. The theoretical description of SSAs has proven to be a challenge [1], since the leading collinear partonic contribution to the asymmetries vanishes [2]. Over the past few years, there have been a number of theoretical developments that have led to much progress in the exploration of the underlying physics for single spin asymmetry phenomena. These developments mainly follow two lines: the so-called transverse momentum dependent (TMD) approach [3, 4, 5, 6, 7, 8, 9, 10], which uses parton distributions and/or fragmentation functions that depend on partonic transverse momentum, and the twist-three quark-gluon correlation function approach [11, 12, 13, 14, 15, 16, 17, 18]. More recently, it has been found in some cases that the two approaches are closely related and describe the same physics [19, 20].

So far, however, phenomenological applications of the approaches have been limited to the "bare" parton model, that is, to the zeroth order of perturbation theory without any QCD corrections, as the latter were generally not available. This situation was remedied very recently when the leading-order ( $\mathrm{LO}$ ) kernels for the scale evolution of the relevant twist-three correlation functions were derived [21, 22]. In this paper, we take a further step toward a more comprehensive QCD description of single-spin phenomena by calculating next-to-leading order (NLO) QCD corrections for a particular observable, the transverse momentum weighted SSA in Drell-Yan lepton pair production.

As demonstrated by many examples, next-to-leading order perturbative QCD (pQCD) corrections are typically very important in hadronic processes. They often lead to significant $K$-factors, and also allow estimates of the size of yet higher order corrections. Moreover, an NLO calculation for a particular physical process will provide a direct test of QCD factorization for the associated observable, complementing the general arguments for such factorizations [7, 8, 23]. One-loop pQCD corrections to the hard-scattering factors in the TMD factorization approach have been calculated for the observables associated with the so-called $k_{\perp}$-even TMD parton distributions [7]. For the related $k_{\perp}$-odd TMD observables, especially relevant for SSAs in various processes, there has not been any particular calcu- 
lation so far. The same is true for the twist-three approach, where all calculations for the SSAs so far have been at leading order only [12, 15, 16]. Previous studies have shown that QCD corrections for higher-twist observables are much more complicated to obtain than for leading-twist ones [24, 25, 26, 27]. This is related both to technical difficulties resulting from more complex partonic states, and to possible mixing between higher-twist matrix elements [24].

On the other hand, the recent developments, especially the consistency between the TMD approach and the twist-three approach found in [19], have provided confidence in our understanding of the underlying theoretical description of single-spin phenomena. They naturally motivate a study of NLO corrections to SSA observables. The calculations and results of [19] will be the starting point for our derivation of the NLO corrections to the SSA in the Drell-Yan process. In this process, a transversely polarized nucleon with momentum $P_{A}$ scatters off an unpolarized nucleon $\left(P_{B}\right)$ to produce a virtual photon with invariant mass $Q$ and transverse momentum $q_{\perp}$, which subsequently decays into a lepton pair,

$$
p_{\uparrow}\left(P_{A}, S_{\perp}\right) p\left(P_{B}\right) \rightarrow \gamma^{*}\left(Q^{2}, q_{\perp}\right)+X \rightarrow \ell^{+} \ell^{-}+X
$$

where $S_{\perp}$ is the transverse polarization vector of the incident nucleon. The Drell-Yan process is bested suited as a first example for the calculation of NLO corrections to single-spin processes. It is among the simplest processes in hadronic scattering, and its single spin asymmetry is also kinematically simpler than those for other processes. For example, for scattering with single transverse polarization, the pair transverse momentum $q_{\perp}$ and the polarization vector $S_{\perp}$ are simply correlated as $\epsilon^{\alpha \beta} S_{\perp}^{\alpha} q_{\perp}^{\beta}=\left|S_{\perp}\right|\left|q_{\perp}\right| \sin \phi$, where $\phi$ is the azimuthal angle of $\vec{q}_{\perp}$ relative to that of $\vec{S}_{\perp}$. If $\vec{q}_{\perp}$ is measured experimentally, the corresponding single spin asymmetry receives contributions from the so-called Sivers effect in the TMD approach, applicable when $q_{\perp} \ll Q$, or from the twist-three Qiu-Sterman matrix elements when $q_{\perp} \sim Q$. As we mentioned above, the two approaches coincide in the kinematic regime of overlap [19]. In the following, we will make use of this fact.

The transverse momentum of the virtual photon (or the lepton pair) generally depends on various transverse momenta in the process, namely those of the initial partons, and those generated by gluon radiation. For the cross section differential in transverse momentum, one has to be careful to classify the different contributions, as a TMD factorization only exists in the limit of small transverse momentum, $q_{\perp} \ll Q[7,19]$. However, if we integrate over all 
transverse momentum $q_{\perp}$, the cross section will depend only on the longitudinal momentum fraction of the virtual photon, and a collinear factorization approach will apply. For the single transverse-spin dependent cross section, we have to suitably weigh with transverse momentum in order to obtain a non-vanishing result, because the unintegrated cross section has linear dependence on $\vec{q}_{\perp}$. The weighted cross section is defined as [28, 29]:

$$
\left\langle q_{\perp} \Delta \sigma\left(S_{\perp}\right)\right\rangle \equiv \int d^{2} q_{\perp}\left|q_{\perp}\right| \sin \phi \frac{d \Delta \sigma\left(S_{\perp}\right)}{d^{2} q_{\perp}},
$$

where we have simplified the expression by omitting dependence on any other kinematic variables. Since the transverse momentum has been integrated out, the above weighted cross section can be properly formulated in the collinear factorization approach [20], and can be factorized into parton distributions and/or twist-three correlation functions for the incident nucleons, and partonic hard-scattering functions. In case of the SSA, the quarkgluon correlation function in the polarized nucleon will be an important ingredient in the factorization formula. It will be part of the following factorization formula for the above $q_{\perp}$-weighted cross section:

$$
\frac{d\left\langle q_{\perp} \Delta \sigma\left(S_{\perp}\right)\right\rangle}{d Q^{2}}=\sigma_{0} \int \frac{d x_{1}}{x_{1}} \frac{d x_{2}}{x_{2}} \frac{d x^{\prime}}{x^{\prime}} T_{F, q}\left(x_{1}, x_{2}\right) \bar{q}\left(x^{\prime}\right) \mathcal{H}\left(x_{1}, x_{2} ; x^{\prime}\right),
$$

where $\sigma_{0}=4 \pi \alpha_{\mathrm{em}}^{2} / 3 N_{C} s Q^{2}$, with $s=\left(P_{A}+P_{B}\right)^{2}, \bar{q}\left(x^{\prime}\right)$ denotes the anti-quark distribution of the unpolarized nucleon, and $T_{F, q}$ the Qiu-Sterman matrix element for quark $q$. We have restricted ourselves here to one quark flavor; extension to more flavors and to scattering off a quark from the unpolarized nucleon is trivial. In the following, we will drop the label $q$ of $T_{F, q}$ for simplicity. As indicated, $T_{F}$ is a function of two separate light-cone variables, and thus the convolution over momentum fraction will include both, as we will see. $T_{F}$ is defined as

$$
\begin{aligned}
T_{F}\left(x_{1}, x_{2}\right) & \equiv \int \frac{d \zeta^{-} d \eta^{-}}{4 \pi} e^{i\left(x_{1} P_{A}^{+} \eta^{-}+\left(x_{2}-x_{1}\right) P_{A}^{+} \zeta^{-}\right)} \\
& \times \epsilon_{\perp}^{\beta \alpha} S_{\perp \beta}\left\langle P_{A}, S\left|\bar{\psi}(0) \mathcal{L}\left(0, \zeta^{-}\right) \gamma^{+} g F_{\alpha}{ }^{+}\left(\zeta^{-}\right) \mathcal{L}\left(\zeta^{-}, \eta^{-}\right) \psi\left(\eta^{-}\right)\right| P_{A}, S\right\rangle
\end{aligned}
$$

where $\mathcal{L}$ is the proper gauge link to make the matrix element gauge invariant, and where the sums over color and spin indices are implicit.

In the factorization formula Eq. (3), the hard-scattering function can be expanded as a series in the strong coupling constant,

$$
\mathcal{H}=\mathcal{H}^{(0)}+\frac{\alpha_{s}}{2 \pi} \mathcal{H}^{(1)}+\cdots,
$$


where $\mathcal{H}^{(0)}$ is the leading order term, $\mathcal{H}^{(1)}$ the NLO one, and so forth. In the following, we will demonstrate that the above factorization formula is valid at NLO level. In particular, the collinear divergence can be factorized into the parton distribution and quark-gluon correlation function, whereas the hard coefficient function is free of any divergence. The real-gluon radiation diagrams have already been studied in [19], and the results can be carried over to our present calculation with relatively little effort. We will compute the virtual corrections as well. It is important to check that the soft divergence in real-gluon radiation is canceled by that in the virtual diagrams, so that we are left with only collinear divergences, which can be absorbed into the parton distribution and/or the twist-three correlation function, where they give rise to the scale evolution of the distributions.

The rest of the paper is organized as follows. In Sec. II, we will derive the leading order expression for the hard coefficient, and calculate the virtual correction at next-to-leading order. In Sec. III, we calculate the real-gluon radiation contributions, and combine them with the virtual corrections. We will show that the soft divergence is canceled in the sum, and that the remaining collinear divergence can be removed by collinear factorization. We conclude our paper in Sec. IV.

\section{BORN DIAGRAMS AND VIRTUAL CORRECTIONS}

At the leading order, the virtual photon is produced in the quark-antiquark annihilation subprocess. In order to obtain a non-vanishing weighted transverse-spin-dependent cross section $\left\langle q_{\perp} \Delta \sigma\left(S_{\perp}\right)\right\rangle$, we have to include an initial state interaction as shown in Fig. 1, which provides the required phase [14]. We perform our calculations in covariant gauge. Let $p^{\prime}$ denote the momentum of the incident anti-quark, $k_{q 1}$ that of the initial quark to the left of the cut, $k_{q 2}$ that on the right, and $k_{g}=k_{q 2}-k_{q 1}$ the momentum of the polarized gluon attaching to the hard part. This attachment may take place on the left side of the cut, as shown in Fig. 1(a), or on the right side, as in 1(b).

The polarized gluon is associated with a gauge potential $A^{\mu}$, and one of the leading contributions comes from its component $A^{+}$. The gluon's momentum is dominated by $x_{g} P+k_{g \perp}$, where $x_{g}$ is the longitudinal momentum fraction with respect to the polarized proton. The transverse momentum $k_{g \perp}$ flows through the perturbative diagram and returns to the polarized proton through the quark lines. The contribution to the single-transverse- 


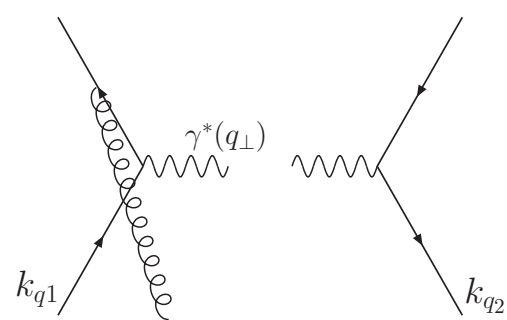

(a)

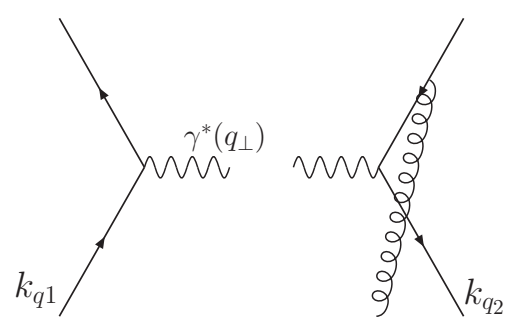

(b)

FIG. 1: Leading order contribution to the weighted transverse spin-dependent cross section.

spin asymmetry arises from terms linear in $k_{g \perp}$ which, when combined with $A^{+}$, yield $\partial^{\perp} A^{+}$, a part of the gauge field strength tensor $F^{\perp+}$. In order to compute this contribution, we expand the partonic scattering amplitudes in terms of $k_{g \perp}$ up to the linear term. The weighted cross section can, in general, be written as

$$
\frac{d\left\langle q_{\perp} \Delta \sigma\left(S_{\perp}\right)\right\rangle}{d Q^{2}}=\frac{\epsilon^{\beta \alpha} S_{\perp}^{\beta}}{2 s} \int \frac{d^{4} k_{q 1}}{(2 \pi)^{4}} \frac{d^{4} k_{q 2}}{(2 \pi)^{4}}\left(q_{\perp}^{\alpha} H\left(k_{q 1}, k_{q 2} ; Q^{2}\right)\right) \bar{q}\left(x^{\prime}\right) T_{a}\left(k_{q 1}, k_{q 2}\right),
$$

where $T_{a}\left(k_{q 1}, k_{q 2}\right)$ represents the non-perturbative matrix element for the polarized nucleon with full momentum dependence on $k_{q 1}$ and $k_{q 2}$. In the above weighted cross section, we have an explicit term $q_{\perp}^{\alpha}$ in the integral, along with the hard partonic part $H\left(k_{q 1}, k_{q 2} ; Q^{2}\right)$.

To obtain the collinear factorization formula Eq. (3), we have to perform a collinear expansion of the hard partonic part. For the leading Born diagrams in Fig. 1, we find that $q_{\perp}$ is related to the transverse momenta of the two quark lines as: $q_{\perp}=k_{q 2 \perp}$ for Fig. 1(a) and $q_{\perp}=k_{q 1 \perp}$ for Fig. 1(b). Therefore, the contribution from Fig. 1(a) to the collinear expansion of Eq. (6) will be

$$
\left.\left(q_{\perp}^{\alpha} H\left(k_{q 1}, k_{q 2} ; Q^{2}\right)\right)\right|_{\text {Fig.1(a) }}=\frac{i g}{-\left(k_{q 2}^{+}-k_{q 1}^{+}\right)-i \epsilon} k_{q 2 \perp}^{\alpha},
$$

where the propagator associated with the initial state interaction produces the pole at $k_{g}^{+}=$ 0. The single spin asymmetry arises from the phase of this pole. Similarly, the contribution from Fig. 1(b) will be

$$
\left(q_{\perp}^{\alpha} H\left(k_{q 1}, k_{q 2} ; Q^{2}\right)\right)_{\text {Fig.1(b) }}=-\frac{i g}{-\left(k_{q 2}^{+}-k_{q 1}^{+}\right)-i \epsilon} k_{q 1 \perp}^{\alpha}
$$

The total contribution is thus

$$
\left(q_{\perp}^{\alpha} H\left(k_{q 1}, k_{q 2} ; Q^{2}\right)\right)_{\text {Fig. } 1(\mathrm{a}+\mathrm{b})}=\frac{i g}{-\left(k_{q 2}^{+}-k_{q 1}^{+}\right)-i \epsilon}\left(k_{q 2 \perp}^{\alpha}-k_{q 1 \perp}^{\alpha}\right)=\frac{i g}{-\left(k_{q 2}^{+}-k_{q 1}^{+}\right)-i \epsilon} k_{g \perp}^{\alpha} .
$$


When integrated over the transverse and light-cone-minus components of the two momenta $k_{q 1}$ and $k_{q 2}$, the combined terms $T_{a}\left(k_{q 1}, k_{q 2}\right)$ and $k_{g \perp}$ produce the matrix element $T_{F}(x, x)$. One then obtains the leading order contribution to the weighted cross section as [28]

$$
\frac{d\left\langle q_{\perp} \Delta \sigma\left(S_{\perp}\right)\right\rangle}{d Q^{2}}=\sigma_{0} \int \frac{d x}{x} \frac{d x^{\prime}}{x^{\prime}} T_{F}(x, x) \bar{q}\left(x^{\prime}\right) \delta\left(1-Q^{2} / x x^{\prime} s\right)
$$

from which one can readily determine the leading order hard coefficient to be

$$
\mathcal{H}\left(x_{1}, x_{2} ; x^{\prime}\right)=\delta\left(1-\frac{x_{2}}{x_{1}}\right) \delta(1-z),
$$

where $z=Q^{2} / \hat{s}$ with $\hat{s}=x_{1} x^{\prime} s$.

The above derivation shows that the Born kinematics greatly simplify the collinear expansion for the hard partonic part, because of momentum conservation. We can utilize this feature in the calculations of the virtual corrections to the Born diagrams as well. At one-loop order, the virtual corrections contain two types of diagrams shown in Fig. 2. In the upper two diagrams $(a, b)$ of Fig. 2 the polarized gluon attaches to the side of the cut opposite from the loop correction which is represented by a blob. On the other hand, in the lower two diagrams $(c, d)$, the gluon attaches to the side that also has the loop. The loops are displayed in detail in Figs. 3 and 4. Fig. 3 is the usual vertex correction, plus self-energy diagrams. If the gluon is on the side of the loop, we have to attach the gluon to all possible places in the virtual diagrams, see Fig. 4.

As before, the Born kinematics simplifies the collinear expansion in the calculations of these diagrams. For example, to obtain the contributions from Figs. 2(a,b), we can simply multiply the Born result in Eq. (9) by the known [30] Drell-Yan virtual correction factor, giving

$$
\left.\left(q_{\perp}^{\alpha} H\left(k_{q 1}, k_{q 2} ; Q^{2}\right)\right)\right|_{\text {Fig. } 2(\mathrm{a}+\mathrm{b})}=\frac{i g}{-\left(k_{q 2}^{+}-k_{q 1}^{+}\right)-i \epsilon} k_{g \perp}^{\alpha} \frac{\alpha_{s}}{4 \pi} C_{F}\left(\frac{4 \pi \mu^{2}}{Q^{2}}\right)^{\epsilon}\left[-\frac{2}{\epsilon^{2}}-\frac{3}{\epsilon}-8+\pi^{2}\right],
$$

where $C_{F}=\left(N_{c}^{2}-1\right) / 2 N_{c}$ with $N_{c}=3$ the number of colors. Here we have used dimensional regularization, with $D=4-2 \epsilon$ space-time dimensions and $\mu$ the mass scale to be introduced in order to keep coupling constants dimensionless ${ }^{1}$. In obtaining this result, it is essential that the one-loop virtual correction in the Drell-Yan process amounts to a simple multiplicative factor to the vertex $\gamma^{\mu}$. As for the Born diagram, the collinear expansion is trivial,

\footnotetext{
${ }^{1}$ Note that we also follow Ref. [30] to absorb a factor $(1-\epsilon)$ in the normalization $\sigma_{0}$, which universally appears in all matrix elements and hence does not affect the final results.
} 

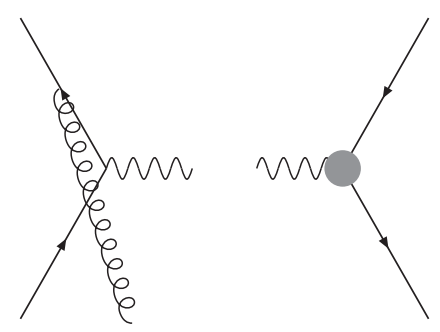

(a)
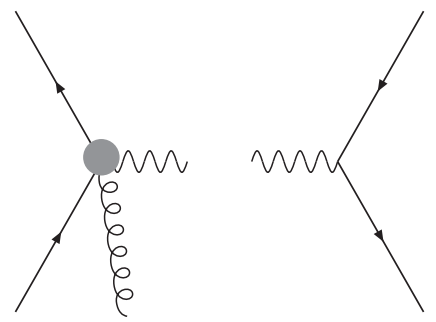

(c)
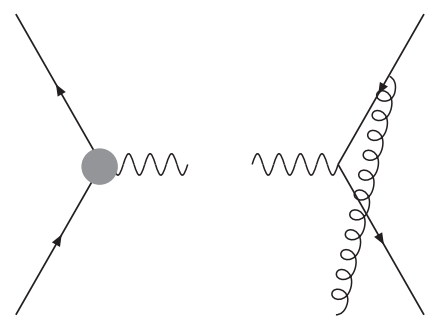

(b)

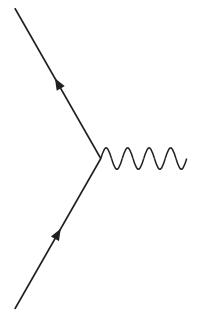

$(d)$

FIG. 2: One-loop virtual correction to the weighted cross section: the gluon attaches to the opposite side of the loop correction (upper two diagrams); the gluon attaches to the same side of the loop corrections (lower two diagrams).
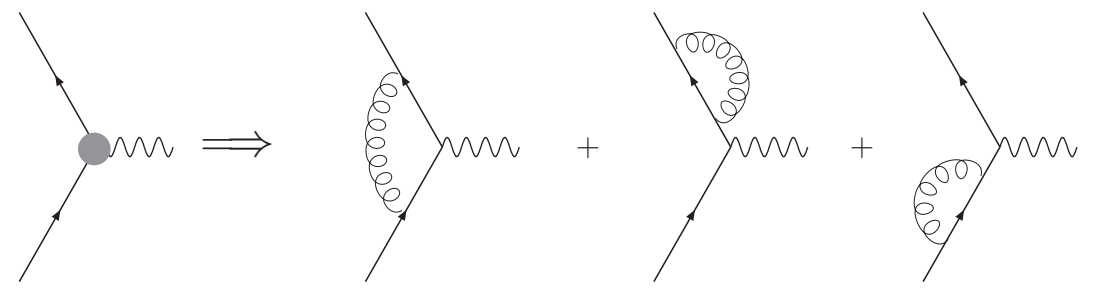

FIG. 3: Corrections to the quark-antiquark-photon vertex, corresponding to the blob in the upper two diagrams of Fig. Q.

and the phase for the SSA comes from the initial state interaction, i.e., the denominator of Eq. (12).

The calculation of Figs. 2(c,d) is more cumbersome, but again the collinear expansion will receive contributions proportional to $k_{q 1 \perp}$ and $k_{q 2 \perp}$ just as in Eqs. (7), (8) . After a lengthy calculation, we find that the diagrams in Fig. 4 lead to the following result for the initial state interaction contribution:

$$
\left.\left(q_{\perp}^{\alpha} H\left(k_{q 1}, k_{q 2} ; Q^{2}\right)\right)\right|_{\text {Fig. } 2(\mathrm{c}+\mathrm{d})}=\frac{i g}{-\left(k_{q 2}^{+}-k_{q 1}^{+}\right)-i \epsilon} k_{g \perp}^{\alpha} \frac{\alpha_{s}}{4 \pi} C_{F}\left(\frac{4 \pi \mu^{2}}{Q^{2}}\right)^{\epsilon}\left[-\frac{2}{\epsilon^{2}}-\frac{3}{\epsilon}-8+\pi^{2}\right],
$$



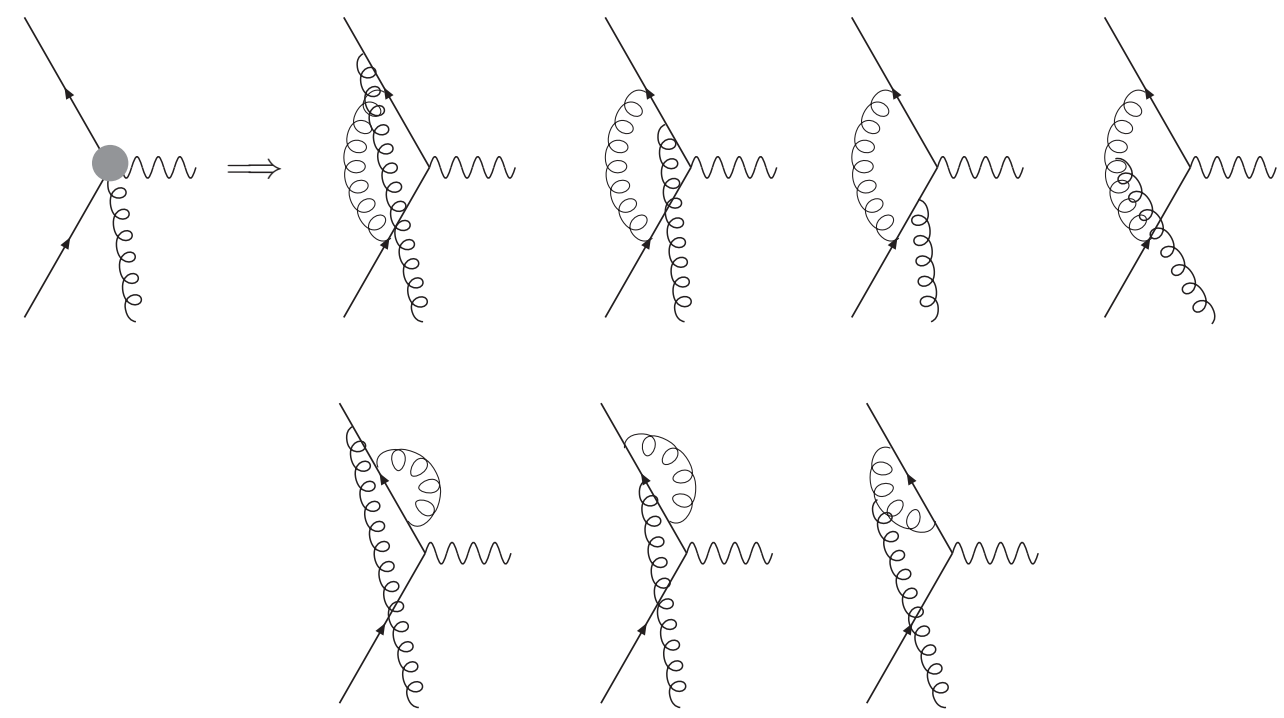

FIG. 4: Corrections to the quark-antiquark-photon vertex with gluon attachment, corresponding to the blob in the lower two diagrams of Fig. Q.

identical to that for Figs. 2(a,b). Substituting the results in Eqs. (12) and (13) into the collinear expansion formula, and accounting for the leading-order phase space in $D$ dimensions, we find the total virtual correction to the weighted single-spin cross section:

$$
\sigma_{0} \frac{\alpha_{s}}{2 \pi} C_{F}\left(\frac{4 \pi \mu^{2}}{Q^{2}}\right)^{\epsilon} \frac{1}{\Gamma(1-\epsilon)} \int \frac{d x}{x} \frac{d x^{\prime}}{x^{\prime}} T_{F}(x, x) \bar{q}\left(x^{\prime}\right) C_{F} \delta(1-z)\left[-\frac{2}{\epsilon^{2}}-\frac{3}{\epsilon}-8+\pi^{2}\right] .
$$

In the next section, we will calculate the real-gluon radiation contribution, and obtain the final result for the NLO correction. It is important to verify that the soft divergence in the above virtual corrections is canceled against that in the real diagrams. We will check this cancelation in the next section.

\section{REAL CORRECTIONS AND FINAL RESULTS}

The real-gluon emission contributions to the single-spin asymmetry for the Drell-Yan process have been computed in [19]. In that paper, the focus was on the SSA at fixed $q_{\perp}$, whereas in our present calculation we are considering the $q_{\perp}$-weighted cross section, which involves integration over all $q_{\perp}$. While we can still use much of the set-up of the calculations of [19], we have to redo them in $D=4-2 \epsilon$ dimensions. This is relatively straightforward. Another issue we need to address is the transverse-momentum flow in the diagrams. In the calculations performed in [19], the virtual photon is an "observed" particle in the sense 
that its momentum is kept fixed in the collinear expansion of the hard partonic scattering amplitudes $H\left(k_{q 1}, k_{q 2} ; Q^{2}\right)$. Since we are integrating over all $q_{\perp}$ in the present calculation, one might think that the photon momentum could be a function of the transverse momenta of the initial partons and hence change the collinear expansion. However, because of momentum conservation it turns out that it is sufficient to allow transverse-momentum flow only through the radiated gluon.

Before going into the calculation, we note that we will only consider contributions associated with the twist-three quark-gluon correlation function. There are two types of such contributions: real-gluon emission in the subprocess $(q g)+\bar{q} \rightarrow \gamma^{*}+g$, and the quark-gluon Compton process $(q g)+g \rightarrow \gamma^{*}+q$. We do not consider in this study contributions entering with a three-gluon twist-three correlation function [16, 31]. We also ignore contributions by the "axial" twist-three quark-gluon correlation function considered in Ref. [32].

The real-gluon radiation diagrams yield soft and collinear divergences when integrated over the transverse momentum, which are regularized by dimensional regularization. Following a standard procedure for the phase space integrals as in the case of the spin-averaged cross section [30], we obtain the following expression for the real-gluon radiation contribution to the transverse momentum weighted spin-dependent cross section:

$$
\begin{aligned}
\frac{d\left\langle q_{\perp} \Delta \sigma\left(S_{\perp}\right)\right\rangle}{d Q^{2}}= & \sigma_{0} \frac{\alpha_{s}}{2 \pi} \int \frac{d x}{x} \frac{d x^{\prime}}{x^{\prime}}\left(\frac{4 \pi \mu^{2}}{Q^{2}}\right)^{\epsilon} \frac{1}{\Gamma(1-\epsilon)} z^{\epsilon}(1-z)^{1-2 \epsilon} \int_{0}^{1} d v(v(1-v))^{-\epsilon} \\
& \left\{x \frac{\partial}{\partial x} T_{F}(x, x)\left(D_{q \bar{q}} \bar{q}\left(x^{\prime}\right)+D_{q g} g\left(x^{\prime}\right)\right)\right. \\
& +T_{F}(x, x)\left(N_{q \bar{q}}^{s} \bar{q}\left(x^{\prime}\right)+N_{q g}^{s} g\left(x^{\prime}\right)\right) \\
& \left.+T_{F}\left(x, x-\bar{x}_{g}\right)\left(N_{q \bar{q}}^{h} \bar{q}\left(x^{\prime}\right)+N_{q g}^{h} g\left(x^{\prime}\right)\right)\right\}
\end{aligned}
$$

where $v$ is related to the partonic center-of-mass scattering angle $\theta$ by $v=(1+\cos \theta) / 2$. The above expression contains three contributions. The first two are the derivative and non-derivative terms from the soft-pole diagrams, respectively; the third is the contribution by hard-pole diagrams, which only have non-derivative pieces. In $D=4-2 \epsilon$ dimensions, 
we obtain the following expressions for the corresponding partonic hard-scattering terms ${ }^{2}$ :

$$
\begin{aligned}
D_{q \bar{q}}= & \frac{1}{2 N_{c}} \frac{-\hat{t}}{\hat{s}}\left[(1-\epsilon)\left(\frac{\hat{u}}{\hat{t}}+\frac{\hat{t}}{\hat{u}}\right)+\frac{2 Q^{2} \hat{s}}{\hat{t} \hat{u}}-2 \epsilon\right], \\
D_{q g}= & -\frac{N_{c}^{2}}{2\left(N_{c}^{2}-1\right)} \frac{-\hat{t}}{\hat{s}}\left[(1-\epsilon)\left(\frac{\hat{s}}{-\hat{t}}+\frac{-\hat{t}}{\hat{s}}\right)-\frac{2 Q^{2} \hat{u}}{\hat{t} \hat{s}}+2 \epsilon\right], \\
N_{q \bar{q}}^{(s)}= & \frac{1}{2 N_{c}} \frac{1}{-\hat{s} \hat{t} \hat{u}}\left[Q^{2}\left(\hat{u}^{2}-\hat{t}^{2}\right)+2 Q^{2} \hat{s}\left(Q^{2}-2 \hat{t}\right)-\left(\hat{u}^{2}+\hat{t}^{2}\right) \hat{t}\right. \\
& \left.+\epsilon\left(\hat{s} \hat{t}^{2}-\hat{s} \hat{u}^{2}+2 \hat{t}^{3}+3 \hat{t}^{2} \hat{u}-\hat{u}^{3}\right)\right], \\
N_{q g}^{(s)}= & \frac{N_{c}^{2}}{2\left(N_{c}^{2}-1\right)} \frac{1}{-\hat{s}^{2} \hat{t}}\left[Q^{2}\left(\hat{s}^{2}-\hat{t}^{2}\right)+2 Q^{2} \hat{u}\left(Q^{2}-2 \hat{t}\right)-\left(\hat{s}^{2}+\hat{t}^{2}\right) \hat{t}\right. \\
& \left.+\epsilon\left(\hat{u} \hat{t}^{2}-\hat{u} \hat{s}^{2}+2 \hat{t}^{3}+3 \hat{t}^{2} \hat{s}-\hat{s}^{3}\right)\right], \\
N_{q \bar{q}}^{(h)}= & \left(\frac{1}{2 N_{c}}+C_{F} \frac{\hat{s}}{\hat{s}+\hat{u}}\right) \frac{\left(Q^{2}-\hat{t}\right)^{3}+Q^{2} \hat{s}^{2}-\epsilon(\hat{s}+\hat{u})(\hat{t}+\hat{u}) \hat{u}}{\hat{s} \hat{t} \hat{u}}, \\
N_{q g}^{(h)}= & \left(\frac{-N_{c}^{2}}{2\left(N_{c}^{2}-1\right)}+T_{R} \frac{\hat{s}}{\hat{s}+\hat{u}}\right) \frac{\left(Q^{2}-\hat{t}\right)^{3}+Q^{2} \hat{u}^{2}-\epsilon(\hat{s}+\hat{u})(\hat{s}+\hat{t}) \hat{s}}{-\hat{s}^{2} \hat{t}},
\end{aligned}
$$

where $\hat{s}, \hat{t}$, and $\hat{u}$ are the partonic Mandelstam variables for the $2 \rightarrow 2$ processes, which can be expressed in terms of $Q^{2}$ and $z$ as

$$
\hat{s}=\frac{Q^{2}}{z}, \hat{t}=-\frac{Q^{2}}{z}(1-z)(1-v), \quad \hat{u}=-\frac{Q^{2}}{z}(1-z) v .
$$

From the above expressions, we see that the integral over $v$ will contain divergences when $v \rightarrow 0$ or $v \rightarrow 1$. The main task in this calculation is to separate these divergences and identify them as soft or collinear, so that they can be canceled appropriately.

First, let us examine the derivative term from the soft-gluon pole contribution in the $q \bar{q}$ channel. After integrating over $v$, it becomes

$$
\begin{gathered}
\sigma_{0} \frac{\alpha_{s}}{2 \pi}\left(\frac{4 \pi \mu^{2}}{Q^{2}}\right)^{\epsilon} \frac{1}{\Gamma(1-\epsilon)} \frac{1}{2 N_{c}} \int \frac{d x}{x} \frac{d x^{\prime}}{x^{\prime}} \bar{q}\left(x^{\prime}\right)\left\{\left(-\frac{1}{\epsilon}\right) T_{F}(x, x) 2 z^{2}+\right. \\
\left.\times\left[x \frac{\partial}{\partial x} T_{F}(x, x)\right]\left(1+z^{2}\right) \ln \frac{(1-z)^{2}}{z}\right\}
\end{gathered}
$$

where we have performed an integration by parts in order to simplify the $1 / \epsilon$ term. The latter comes from a collinear divergence, which will be canceled by factorization into the evolved $T_{F}$ function. There is no soft divergence in this term, which is expected because the virtual diagrams do not contribute to the derivative terms, as we saw earlier.

\footnotetext{
${ }^{2}$ Note that in contrast to [30] we average over the polarizations of the initial gluon in the $q g$ subprocess by the factor $1 /(2(1-\epsilon))$, as is customary in the $\overline{\mathrm{MS}}$ scheme.
} 
The soft-pole derivative terms in the $q g$ channel give only finite contributions. It is easy to perform the phase space integration, and we obtain

$$
\sigma_{0} \frac{\alpha_{s}}{2 \pi} \frac{-N_{c}^{2}}{2\left(N_{c}^{2}-1\right)} \int \frac{d x}{x} \frac{d x^{\prime}}{x^{\prime}}\left[x \frac{\partial}{\partial x} T_{F}(x, x)\right] g\left(x^{\prime}\right)\left(1+z^{2}\right)\left[\frac{1}{3}(1-z)\left(4+4 z^{2}-5 z\right)\right] .
$$

The non-derivative term from the soft-pole diagrams in the $q \bar{q}$ channel has both soft and collinear divergences. After integrating over the phase space variable $v$, we get

$$
\begin{aligned}
& \sigma_{0} \frac{\alpha_{s}}{2 \pi}\left(\frac{4 \pi \mu^{2}}{Q^{2}}\right)^{\epsilon} \frac{1}{\Gamma(1-\epsilon)} \frac{1}{2 N_{c}} \int \frac{d x}{x} \frac{d x^{\prime}}{x^{\prime}} T_{F}(x, x) \bar{q}\left(x^{\prime}\right)\left\{-\frac{2}{\epsilon^{2}} \delta(1-z)-\frac{1}{\epsilon} \frac{z^{3}-3 z^{2}-z-1}{(1-z)_{+}}\right. \\
& \left.+\frac{\pi^{2}}{3} \delta(1-z)+\left(z^{3}-3 z^{2}-z-1\right)\left[2\left(\frac{\ln (1-z)}{1-z}\right)_{+}+\frac{\ln z}{1-z}\right]\right\} .
\end{aligned}
$$

The double-pole $1 / \epsilon^{2}$ term represents a soft-collinear divergence, which will eventually be canceled. The collinear-divergent term $\propto 1 / \epsilon$ will generate part of the splitting function for the evolution of the unpolarized quark distribution and/or the twist-three correlation function.

Now we turn to the hard-pole contributions, which are only non-derivative. For these contributions, the two arguments in the twist-three quark-gluon correlation function are different and may depend on partonic kinematics. As a result, the $v$-integral is somewhat more involved, and for some parts the integral cannot be performed completely. First, we will separate these parts by introducing "plus"-distributions of the form

$$
\int_{0}^{1} d v \frac{g(v)}{v_{+}} \equiv \int d v \frac{g(v)-g(0)}{v}, \quad \int_{0}^{1} d v \frac{g(v)}{(1-v)_{+}} \equiv \int d v \frac{g(v)-g(1)}{1-v},
$$

in the integrand. The distributions arise from terms $\propto v^{-1},(1-v)^{-1}$ in the integrand which, when combined with the phase space factor $(v(1-v))^{-\epsilon}$, give rise to identities of the form

$$
v^{-1-\epsilon}=-\frac{1}{\epsilon} \delta(v)+\frac{1}{(v)_{+}}-\epsilon\left(\frac{\ln (v)}{v}\right)_{+}+\mathcal{O}\left(\epsilon^{2}\right) .
$$

For the $q \bar{q}$ channel, the part that cannot be further integrated over $v$ analytically then reads:

$$
\begin{aligned}
& \sigma_{0} \frac{\alpha_{s}}{2 \pi} \int \frac{d x}{x} \frac{d x^{\prime}}{x^{\prime}} \frac{d v}{1-z}\left(\frac{1}{v_{+}}+\frac{1}{(1-v)_{+}}\right) T_{F}\left(x, x \frac{z}{1-v(1-z)}\right) \bar{q}\left(x^{\prime}\right) \\
& \quad \times\left[(1-v(1-z))^{3}+z\right]\left(\frac{1}{2 N_{c}}+C_{F} \frac{1}{1-v(1-z)}\right) .
\end{aligned}
$$

Both plus distributions are needed because the integrand is divergent at both $v \rightarrow 0$ and $v \rightarrow 1$. The distributions guarantee that the integral over $v$ is finite. Besides, as one can see, 
there is no divergence in the limit $z \rightarrow 1$. The remaining part of the hard-pole contribution in the $q \bar{q}$ channel is then rather straightforward to obtain, and we obtain upon integration over $v$

$$
\begin{aligned}
& \sigma_{0} \frac{\alpha_{s}}{2 \pi}\left(\frac{4 \pi \mu^{2}}{Q^{2}}\right)^{\epsilon} \frac{1}{\Gamma(1-\epsilon)} \int \frac{d x}{x} \frac{d x^{\prime}}{x^{\prime}} \bar{q}\left(x^{\prime}\right)\left\{T _ { F } ( x , x z ) ( \frac { 1 } { 2 N _ { c } } + C _ { F } ) \left[\frac{1}{\epsilon^{2}} \delta(1-z)\right.\right. \\
& \left.\quad-\frac{1}{\epsilon} \frac{1+z}{(1-z)_{+}}-\frac{\pi^{2}}{6} \delta(1-z)+(1+z)\left(2\left(\frac{\ln (1-z)}{1-z}\right)_{+}+\frac{\ln z}{1-z}\right)\right] \\
& +T_{F}(x, x)\left(\frac{1}{2 N_{c}} z+C_{F}\right)\left[\frac{1}{\epsilon^{2}} \delta(1-z)-\frac{1}{\epsilon} \frac{1+z^{2}}{(1-z)_{+}}-\frac{\pi^{2}}{6} \delta(1-z)\right. \\
& \left.\left.+\left(1+z^{2}\right)\left(2\left(\frac{\ln (1-z)}{1-z}\right)_{+}+\frac{\ln z}{1-z}\right)+(1-z)\right]\right\} .
\end{aligned}
$$

Again, we have both soft and collinear divergences.

Similarly, for the hard-pole contribution in the $q g$ channel, we have a regularized part that cannot be further integrated over $v$ analytically:

$$
\begin{aligned}
& \sigma_{0} \frac{\alpha_{s}}{2 \pi} \int \frac{d x}{x} \frac{d x^{\prime}}{x^{\prime}} \frac{d v}{(1-v)_{+}} T_{F}\left(x, x \frac{z}{1-v(1-z)}\right) g\left(x^{\prime}\right) \\
& \quad \times\left[(1-v(1-z))^{3}+v^{2}(1-z)^{2} z\right]\left(\frac{-N_{c}^{2}}{2\left(N_{c}^{2}-1\right)}+T_{R} \frac{1}{1-v(1-z)}\right),
\end{aligned}
$$

and a remaining singular part:

$$
\begin{aligned}
\sigma_{0} \frac{\alpha_{s}}{2 \pi} & \left(\frac{4 \pi \mu^{2}}{Q^{2}}\right)^{\epsilon} \frac{1}{(1-\epsilon) \Gamma(1-\epsilon)} \int \frac{d x}{x} \frac{d x^{\prime}}{x^{\prime}} T_{F}(x, x) g\left(x^{\prime}\right)\left(\frac{-N_{c}^{2}}{2\left(N_{c}^{2}-1\right)} z+T_{R}\right) \\
\times & {\left[-\frac{1}{\epsilon}\left(z^{2}+(1-z)^{2}\right)+\left(z^{2}+(1-z)^{2}\right) \ln \frac{(1-z)^{2}}{z}+1\right] . }
\end{aligned}
$$

There is no soft divergence. The collinear divergence will be canceled by factorization of the gluon splitting contribution to the spin-averaged anti-quark distribution function.

As we mentioned at the beginning, the soft divergence has to disappear after adding the contributions by the real-gluon radiation and virtual diagrams. Indeed this happens, as inspection of Eqs. (14) and (25), (29) shows. Specifically, the term $\propto 1 / \epsilon^{2}$ from the softpole diagrams cancels that from the hard-pole diagrams associated with color-factor $1 / 2 N_{c}$, and the remaining $1 / \epsilon^{2}$ term from the hard-pole diagrams (associated with the color-factor $C_{F}$ ) cancels against that from the virtual diagrams. This is an important cross-check on the consistency of our calculations, and demonstrates the importance of the hard-pole diagrams.

After cancelation of soft poles, the result will only contain collinear divergences. We find 
for the remaining pole term

$$
\begin{aligned}
& \sigma_{0} \frac{\alpha_{s}}{2 \pi} \int \frac{d x}{x} \frac{d x^{\prime}}{x^{\prime}}\left(-\frac{1}{\epsilon}\right)\left\{T_{F}(x, x) \bar{q}\left(x^{\prime}\right)\left[2 C_{F}\left(\frac{1+z^{2}}{1-z}\right)_{+}+\left(C_{F}+\frac{1}{2 N_{c}}\right) z\right]\right. \\
& +\left(T_{F}(x, x z)-T_{F}(x, x)\right) \bar{q}\left(x^{\prime}\right)\left(C_{F}+\frac{1}{2 N_{c}}\right) \frac{1+z}{1-z} \\
& \left.+T_{F}(x, x) g\left(x^{\prime}\right) T_{R}\left(z^{2}+(1-z)^{2}\right)\right\} .
\end{aligned}
$$

The residue of this collinear divergence contains the splitting functions governing the evolution of the anti-quark distribution in the unpolarized nucleon and the twist-three correlation function. For the former, we have

$$
\bar{q}(x)=\bar{q}^{(0)}(x)+\frac{\alpha_{s}}{2 \pi} \int \frac{d x^{\prime}}{x^{\prime}}\left(-\frac{1}{\epsilon}\right)\left[\bar{q}\left(x^{\prime}\right) C_{F}\left(\frac{1+z^{2}}{1-z}\right)_{+}+g\left(x^{\prime}\right) T_{R}\left(z^{2}+(1-z)^{2}\right)\right],
$$

where $z=x / x^{\prime}$ and $\bar{q}^{(0)}(x)$ denotes the "bare" leading order anti-quark distribution. Similarly, we obtain the collinear QCD correction to the Qiu-Sterman matrix element at equal momentum fractions:

$$
\begin{aligned}
T_{F}(x, x)= & T_{F}^{(0)}(x, x)+\frac{\alpha_{s}}{2 \pi} \int \frac{d x^{\prime}}{x^{\prime}}\left(-\frac{1}{\epsilon}\right)\left\{T_{F}\left(x^{\prime}, x^{\prime}\right) C_{F}\left(\frac{1+z^{2}}{1-z}\right)_{+}\right. \\
& \left.+\left(C_{F}+\frac{1}{2 N_{C}}\right)\left[\frac{1+z}{1-z} T_{F}\left(x^{\prime}, x^{\prime} z\right)-\frac{1+z^{2}}{1-z} T_{F}\left(x^{\prime}, x^{\prime}\right)\right]\right\} .
\end{aligned}
$$

From this equation, we directly read off the scale evolution equation for the "diagonal" twist-three quark-gluon correlation function at $x_{1}=x_{2}=x$ :

$$
\begin{aligned}
\frac{\partial}{\partial \ln \mu^{2}} T_{F}\left(x, x ; \mu^{2}\right)= & \frac{\alpha_{s}\left(\mu^{2}\right)}{2 \pi} \int \frac{d x^{\prime}}{x^{\prime}}\left\{C_{F} T_{F}\left(x^{\prime}, x^{\prime} ; \mu^{2}\right)\left(\frac{1+z^{2}}{1-z}\right)_{+}\right. \\
& \left.+\frac{N_{c}}{2}\left[T_{F}\left(x^{\prime}, x^{\prime} ; \mu^{2}\right) z-\left(T_{F}\left(x^{\prime}, x^{\prime} ; \mu^{2}\right)-T_{F}\left(x^{\prime}, x^{\prime} z ; \mu^{2}\right)\right) \frac{1+z}{1-z}\right]\right\} \\
& \equiv \frac{\alpha_{s}\left(\mu^{2}\right)}{2 \pi} \int \frac{d x^{\prime}}{x^{\prime}} \mathcal{P}_{q g \rightarrow q g} \otimes T_{F}\left(x^{\prime}, x^{\prime} ; \mu^{2}\right)
\end{aligned}
$$

This evolution equation could also have been obtained from the perturbative calculation of the quark Sivers function at large transverse momentum performed in [19]. However, we note that in that paper a boundary term $(\propto \delta(1-z))$ in the derivative contribution was overlooked ${ }^{3}$. After correcting for this term, the result of [19] becomes consistent with that given above. Eq. (35) is also consistent with the results derived recently by different

\footnotetext{
${ }^{3}$ This term does not, however, affect the consistency of the twist-three and the TMD approaches established in 19].
} 
methods [21, 22]. We note, however, that the evolution equations derived in [21] go beyond ours, as they also contain the contributions from additional operators such as three-gluon ones, which we are not considering here.

A few comments on the evolution equation are in order. First, it is evident that the scale evolution of the "diagonal" $\left(x_{1}=x_{2}=x\right)$ function mixes with the function at $x_{1} \neq x_{2}$, implying that the equation as it stands is not closed. In other words, there will be a more general evolution equation for the full function $T_{F}\left(x_{1}, x_{2}\right)$. This feature is quite general for higher-twist parton distributions and fragmentation functions [24, 25, 26, 27]. Second, there is no particular simplification of the evolution equation in the large- $N_{c}$ limit. This is different from what was discovered for the evolution equations for other twist-three quark distributions, such as $h_{L}(x)$ and $e(x)$, where the evolution equations are closed (diagonal) in that limit [25]. However, we notice that the high- $x$ part of the evolution equation, i.e., the large $z$ limit of the kernel in the integrand, is the same as that for the spin-averaged leading-twist quark distribution, because the term $C_{F}\left(\left(1+z^{2}\right) /(1-z)\right)_{+}$is the ordinary leading order quark splitting function. This property will have important phenomenological consequences [21] for the behavior of the quark-gluon correlation function at high $x$ and thus for SSAs in hadronic processes at forward angles.

We note that the renormalization and evolution of general twist-three quark-gluon operators has been extensively studied over the past two decades [24, 25, 26, 27]. The above result for the evolution of the Qiu-Sterman matrix element should likely also be reproduced from the evolution equations discussed in some of these papers. However, we notice that the QiuSterman matrix element corresponds to a very different projection of the general twist-three quark-gluon correlation function, and we do not expect that there will be a simple relation between the above evolution equation and those for other specific twist-three distributions such as $g_{T}(x)$. The comparison between the above results (or the ones of [21, 22]) and those in [24, 25, 26, 27] is very important and will be addressed in the future.

After $\overline{\mathrm{MS}}$ subtraction of the collinear divergences into the quark-gluon correlation function of the polarized nucleon and the anti-quark distribution of the unpolarized nucleon, we obtain the full NLO expression for the soft-gluon and hard-pole contributions ${ }^{4}$ to the transverse-

\footnotetext{
${ }^{4}$ We remind the reader that we do not consider contributions associated with soft-fermion poles or with a three-gluon twist-three correlation function.
} 
momentum weighted single-spin dependent cross section in Drell-Yan lepton pair production in $p p$ collisions:

$$
\begin{aligned}
\left.\frac{d\left\langle q_{\perp} \Delta\right.}{d Q^{2}}\left(S_{\perp}\right)\right\rangle & =\sigma_{0} \int \frac{d x}{x} \frac{d x^{\prime}}{x^{\prime}} T_{F}\left(x, x ; \mu^{2}\right) \bar{q}\left(x^{\prime} ; \mu^{2}\right) \\
& +\sigma_{0} \frac{\alpha_{s}}{2 \pi} \int \frac{d x}{x} \frac{d x^{\prime}}{x^{\prime}}\left\{\overline { q } ( x ^ { \prime } ; \mu ^ { 2 } ) \left[\ln \frac{Q^{2}}{\mu^{2}}\left(C_{F} \mathcal{P}_{q q}+\mathcal{P}_{q g \rightarrow q g} \otimes T_{F}\left(x, x z ; \mu^{2}\right)\right)\right.\right. \\
& +\frac{1}{2 N_{c}}\left(x \frac{\partial}{\partial x} T_{F}\left(x, x ; \mu^{2}\right)\right)\left(1+z^{2}\right) \ln \frac{(1-z)^{2}}{z}+\left(2\left(\frac{\ln (1-z)}{1-z}\right)_{+}-\frac{\ln z}{1-z}\right) \\
& \times\left(\left(C_{F}\left(1+z^{2}\right)+\frac{2 z^{3}-3 z^{2}-1}{2 N_{c}}\right) T_{F}\left(x, x ; \mu^{2}\right)+\left(\frac{1}{2 N_{c}}+C_{F}\right)(1+z) T_{F}\left(x, x z ; \mu^{2}\right)\right) \\
& \left.+T_{F}\left(x, x ; \mu^{2}\right)\left(\left(C_{F}+\frac{z}{2 N_{c}}\right)(1-z)+C_{F}\left(\frac{2 \pi^{2}}{3}-8\right) \delta(1-z)\right)\right] \\
& +g\left(x^{\prime} ; \mu^{2}\right)\left[\left(x \frac{\partial}{\partial x} T_{F}\left(x, x ; \mu^{2}\right)\right)\left(\frac{-N_{c}^{2}}{2\left(N_{c}^{2}-1\right)}\right) \frac{1}{3}(1-z)\left(4+4 z^{2}-5 z\right)\right. \\
& +T_{F}\left(x, x ; \mu^{2}\right) T_{R}\left(\left(z^{2}+(1-z)^{2}\right) \ln \frac{Q^{2}}{\mu^{2}} \frac{(1-z)^{2}}{z}+2 z(1-z)\right) \\
& \left.\left.+T_{F}\left(x, x ; \mu^{2}\right) \frac{N_{c}^{2}}{2\left(N_{c}^{2}-1\right)} \frac{1}{6}\left(8-27 z+48 z^{2}-29 z^{3}\right)\right]\right\} \\
& +\int \frac{d v}{1-z}\left(\frac{1}{v_{+}}+\frac{1}{(1-v)_{+}}\right) T_{F}\left(x, x \frac{z}{1-v(1-z)} ; \mu^{2}\right) \bar{q}\left(x^{\prime} ; \mu^{2}\right) \\
& +\left[(1-v(1-z))^{3}+z\right]\left(\frac{1}{2 N_{c}}+C_{F} \frac{1}{1-v(1-z)}\right) \\
& +\int \frac{d v}{(1-v)_{+}} T_{F}\left(x, x \frac{z}{1-v(1-z)} ; \mu^{2}\right) g\left(x^{\prime} ; \mu^{2}\right)\left[(1-v(1-z))^{3}+v^{2}(1-z)^{2} z\right] \\
& \times\left(\frac{-N_{c}^{2}}{2\left(N_{c}^{2}-1\right)}+T_{R} \frac{1}{1-v(1-z)}\right) \\
& =(37)
\end{aligned}
$$

As expected, the logarithms containing the factorization scale enter with the splitting functions for the evolution of the twist-three quark-gluon correlation function and the twist-two anti-quark distribution.

One important feature of this result is its behavior near "partonic threshold", that is in the large- $z$ limit of the integrand, corresponding to $\hat{s} \sim Q^{2}$, when the initial partons have "just enough" energy to produce the virtual photon. Setting the scale $\mu=Q$, we have the following structure of the NLO correction in this case:

$$
\frac{d\left\langle q_{\perp} \Delta \sigma\left(S_{\perp}\right)\right\rangle}{d Q^{2}}=\sigma_{0} \frac{\alpha_{s}}{2 \pi} \int \frac{d x}{x} \frac{d x^{\prime}}{x^{\prime}} T_{F}\left(x, x ; \mu^{2}\right) \bar{q}\left(x^{\prime} ; \mu^{2}\right)\left[8 C_{F}\left(\frac{\ln (1-z)}{1-z}\right)_{+}+\ldots\right] .
$$

Here we have written out the "double-logarithmic" term which dominates near threshold in 
the $\overline{\mathrm{MS}}$ scheme. The ellipses denote terms that are subleading relative to this term. The structure of this expression is identical to that for the spin-averaged $q_{\perp}$-integrated NLO cross section near threshold,

$$
\frac{d \sigma}{d Q^{2}}=\sigma_{0} \frac{\alpha_{s}}{2 \pi} \int \frac{d x}{x} \frac{d x^{\prime}}{x^{\prime}} q\left(x ; \mu^{2}\right) \bar{q}\left(x^{\prime} ; \mu^{2}\right)\left[8 C_{F}\left(\frac{\ln (1-z)}{1-z}\right)_{+}+\ldots\right] .
$$

This means that the soft gluon contribution is spin-independent. It contributes in the same way to the spin-averaged and single-spin-dependent cross sections, and will lead to the same soft-gluon threshold resummation effects to these cross sections, at least at the leading double logarithmic level. This observation is very similar to that made for the transverse momentum resummation in the Drell-Yan process [33].

\section{CONCLUSIONS}

In summary, we have derived the NLO perturbative-QCD correction to the transverse momentum weighted single spin asymmetry in Drell-Yan lepton pair production in hadronic collisions. In the calculation, we have shown that the collinear divergences can be absorbed into the NLO twist-three quark-gluon correlation function of the transversely polarized nucleon and the unpolarized quark distribution of the unpolarized nucleon. This procedure also determines the evolution equation for the "diagonal part" of the twist-three Qiu-Sterman matrix element at equal momentum fractions, $x_{1}=x_{2}$. We have found this equation to be consistent with the more complete one derived recently in Ref. [21, 22].

Our calculations suggest that a general factorization formula (see Eq. (3)) exists for the transverse momentum weighted spin-dependent cross section in the Drell-Yan process, in extension of the general factorization arguments given in [23].

We have found that both the evolution kernel and the full NLO expression for the spindependent cross section become identical to their spin-averaged counterparts in the "threshold" limit $\hat{s} \rightarrow Q^{2}$, or $z \rightarrow 1$. This will likely have the phenomenological consequence that the single-spin asymmetry for the Drell-Yan process will be quite stable under NLO corrections, in particular when $\tau=Q^{2} / s$ is large.

It will be important to carry out further studies. We have mentioned already that it may be possible to derive the evolution of the twist-three correlation functions also from some of the results of [24]. Also, it will be important to derive NLO corrections also for 
other processes. For example, extension to semi-inclusive deep inelastic scattering should be relatively straightforward to do.

\section{Acknowledgments}

We thank Zhongbo Kang, Jianwei Qiu, and Jian Zhou for useful comments and valuable discussions. W.V. is grateful to V. Braun, M. Diehl, and D. Müller for useful discussions. This work was supported in part by the U.S. Department of Energy under grant contract DE-AC02-05CH11231. F.Y. and W.V. thank RIKEN, Brookhaven National Laboratory and the U.S. Department of Energy (contract number DE-AC02-98CH10886) for providing the facilities essential for the completion of their work.

[1] M. Anselmino, A. Efremov and E. Leader, Phys. Rept. 261, 1 (1995) [Erratum-ibid. 281, 399 (1997)]; Z. t. Liang and C. Boros, Int. J. Mod. Phys. A 15, 927 (2000); V. Barone, A. Drago and P. G. Ratcliffe, Phys. Rept. 359, 1 (2002).

[2] G. L. Kane, J. Pumplin and W. Repko, Phys. Rev. Lett. 41, 1689 (1978).

[3] S. J. Brodsky, D. S. Hwang and I. Schmidt, Phys. Lett. B 530, 99 (2002); Nucl. Phys. B 642, $344(2002)$.

[4] J. C. Collins, Phys. Lett. B 536, 43 (2002).

[5] X. Ji and F. Yuan, Phys. Lett. B 543, 66 (2002); A. V. Belitsky, X. Ji and F. Yuan, Nucl. Phys. B 656, 165 (2003).

[6] D. Boer, P. J. Mulders and F. Pijlman, Nucl. Phys. B 667, 201 (2003).

[7] X. Ji, J. P. Ma and F. Yuan, Phys. Rev. D 71, 034005 (2005); Phys. Lett. B 597, 299 (2004).

[8] J. C. Collins and A. Metz, Phys. Rev. Lett. 93, 252001 (2004).

[9] D. W. Sivers, Phys. Rev. D 43, 261 (1991).

[10] M. Anselmino, M. Boglione and F. Murgia, Phys. Lett. B 362, 164 (1995); M. Anselmino and F. Murgia, Phys. Lett. B 442, 470 (1998); M. Anselmino, M. Boglione, U. D’Alesio, E. Leader and F. Murgia, Phys. Rev. D 71, 014002 (2005).

[11] A. V. Efremov and O. V. Teryaev, Sov. J. Nucl. Phys. 36, 140 (1982) [Yad. Fiz. 36, 242 (1982)]; A. V. Efremov and O. V. Teryaev, Phys. Lett. B 150, 383 (1985). 
[12] J.W. Qiu and G. Sterman, Phys. Rev. Lett. 67, 2264 (1991); Nucl. Phys. B 378, 52 (1992); Phys. Rev. D 59, 014004 (1998).

[13] Y. Kanazawa and Y. Koike, Phys. Lett. B 478, 121 (2000); Phys. Rev. D 64, 034019 (2001).

[14] D. Boer and J. W. Qiu, Phys. Rev. D 65, 034008 (2002).

[15] C. Kouvaris, J. W. Qiu, W. Vogelsang and F. Yuan, Phys. Rev. D 74, 114013 (2006).

[16] Z. B. Kang, J. W. Qiu, W. Vogelsang and F. Yuan, Phys. Rev. D 78, 114013 (2008).

[17] H. Eguchi, Y. Koike and K. Tanaka, Nucl. Phys. B 763, 198 (2007).

[18] Y. Koike and T. Tomita, arXiv:0903.1923 [hep-ph].

[19] X. Ji, J. W. Qiu, W. Vogelsang and F. Yuan, Phys. Rev. Lett. 97, 082002 (2006); Phys. Rev. D 73, 094017 (2006); Phys. Lett. B 638, 178 (2006).

[20] A. Bacchetta, D. Boer, M. Diehl and P. J. Mulders, JHEP 0808, 023 (2008).

[21] Z. B. Kang and J. W. Qiu, Phys. Rev. D 79, 016003 (2009).

[22] J. Zhou, F. Yuan and Z. T. Liang, arXiv:0812.4484 [hep-ph].

[23] J. W. Qiu and G. Sterman, Nucl. Phys. B 353, 105 (1991); Nucl. Phys. B 353, 137 (1991).

[24] I. I. Balitsky and V. M. Braun, Nucl. Phys. B 311, 541 (1989); P. G. Ratcliffe, Nucl. Phys. B 264, 493 (1986); X. D. Ji and C. h. Chou, Phys. Rev. D 42, 3637 (1990).

[25] A. Ali, V. M. Braun and G. Hiller, Phys. Lett. B 266, 117 (1991); J. Kodaira, Y. Yasui, K. Tanaka and T. Uematsu, Phys. Lett. B 387, 855 (1996); I. I. Balitsky, V. M. Braun, Y. Koike and K. Tanaka, Phys. Rev. Lett. 77, 3078 (1996); D. Müller, Phys. Lett. B 407, 314 (1997); V. M. Braun, G. P. Korchemsky and A. N. Manashov, Phys. Lett. B 476, 455 (2000).

[26] A. V. Belitsky and D. Mueller, Nucl. Phys. B 503, 279 (1997); A. V. Belitsky, Nucl. Phys. B 558, 259 (1999); Nucl. Phys. B 574, 407 (2000).

[27] A. V. Belitsky, X. D. Ji, W. Lu and J. Osborne, Phys. Rev. D 63, 094012 (2001); A. A. Henneman, D. Boer and P. J. Mulders, Nucl. Phys. B 620, 331 (2002).

[28] D. Boer and P. J. Mulders, Phys. Rev. D 57, 5780 (1998).

[29] D. Boer, Phys. Rev. D 60, 014012 (1999).

[30] G. Altarelli, R. K. Ellis and G. Martinelli, Nucl. Phys. B 157, 461 (1979).

[31] X. D. Ji, Phys. Lett. B 289, 137 (1992); Z. B. Kang and J. W. Qiu, Phys. Rev. D 78, 034005 (2008).

[32] Y. Koike, W. Vogelsang and F. Yuan, Phys. Lett. B 659, 878 (2008).

[33] A. Idilbi, X. d. Ji, J. P. Ma and F. Yuan, Phys. Rev. D 70, 074021 (2004). 